Visc Med 33 | 1 | 17

Chronisch-entzündliche Darmerkrankungen

\section{Mit TNF-alpha-Inhibitoren bessere Chancen auf anhaltende Remission}

Die Induktions- und Erhaltungstherapie chronisch-entzündlicher Darmerkrankungen (CED) ist mit den Tumornekrosefaktor (TNF)-alphaInhibitoren so effektiv geworden, dass viele $\mathrm{Pa}$ tienten langfristige klinische und endoskopische Remissionen erreichen können. Deshalb setzt man heute vermehrt auf eine akzelerierte Stepup-Therapie.

Wie Prof. Dr. med. Axel Dignass, Frankfurt, auf dem 10. TNF-alpha-Forum in München ausführte, wurde erst im Verlauf des klinischen Einsatzes der TNF-alpha-Blocker aufgeklärt, warum diese Substanzen so gut wirken. Sie neutralisieren freies und membran- oder rezeptorgebundenes TNF-alpha, weisen aber auch immunologische Mechanismen auf wie die Verbesserung der Barrierefunktion und die Induktion von Immunmodulation. Dazu kommen molekulare Mechanismen wie die Induktion von Apoptose, Zytokinen oder MAP-Kinasen.

Davon profitieren auch CED-Patienten. Sie haben heute auch bei schwerer Erkrankung deutlich bessere Chancen, eine anhaltende Mukosaheilung zu erreichen. Beispielhaft führte Prof. Dr. med. Walter Reinisch, Wien, die PURSUIT-Studie an, in der $42,3 \%$ der Patienten mit moderater bis schwer aktiver Colitis ulcerosa durch eine Induktionstherapie mit 200/100 mg Golimumab innerhalb von 6 Wochen eine Mukosaheilung erzielten, verglichen mit 28,7\% der Patienten in der PlaceboGruppe [1]. Besonders hohe endoskopische Heilungsraten von $78,3 \%$ bzw. $66,4 \%$ fielen bei Patienten auf, deren Stuhlfrequenz sich bereits innerhalb von 6 Wochen normalisiert hatte bzw. die nach diesem Zeitraum keine rektalen Blutungen mehr aufwiesen [2].

Patienten, die auf die Induktionstherapie mit Golimumab angesprochen hatten, nahmen auch an der Erhaltungstherapie-Studie teil [3]. Bis Woche 30 und 54 erzielten $23,2 \%$ der Patienten mit 50 mg Golimumab und 27,8\% mit 100 mg Golimumab eine anhaltende Remission, verglichen mit 15,6\% in der PlaceboGruppe. Eine mukosale Heilung zu beiden Zeitpunkten beobachtete man bei $41,7 \%$ und $42,4 \%$ versus $26,6 \%$ der Patienten.

Der primäre Endpunkt der Studie war jedoch das kontinuierliche klinische Ansprechen. Alle 4 Wochen wurde der klinische MayoSubscore erhoben und zu Woche 30 und 54 der volle Mayo-Score einschließlich Endoskopie. Patienten, die zu irgendeinem Zeitpunkt die Kriterien für klinisches Ansprechen nicht erfüllten, zählten nicht mehr positiv mit beim primären Endpunkt. Den Endpunkt, der also keine Momentaufnahme repräsentiert, sondern die gesamte Therapiedauer von 54 Wochen, erreichten mit Golimumab signifikant mehr Patienten als mit Placebo: $47 \%$ mit 50 mg Golimumab, 49,7\% mit 100 mg Golimumab und $31,2 \%$ mit Placebo.

In Post-hoc-Analysen [4] zeigte sich, dass $67,1 \%$ der Patienten, die die Kriterien einer kontinuierlichen klinischen Response erfüllten, am Ende der Studie auch in klinischer Remission waren. Und 90,4\% dieser Patienten wiesen eine Mukosaheilung auf. Dagegen waren fast keine Patienten ohne kontinuierliche klinische Response am Ende der Studie in Remission oder hatten eine Mukosaheilung erreicht. Diese Daten sprechen laut Reinisch dafür, dass für eine langfristig erfolgreiche Therapie eine regelmäßige engmaschige standardisierte Kontrolle der klinischen Symptome notwendig ist. Aktuelle Langzeitdaten aus der PURSUIT-Studie zeigen, dass eine steroidfreie Remission bei mehr als $80 \%$ der Patienten für bis zu 4 Jahre erhalten blieb [5].
Individualisierte Therapie

Wie PD Dr. med. Carsten Schmidt, Jena, betonte, hat auch die individualisierte Therapie schon längst Einzug in die Behandlung von CED gehalten. Als Beispiele nannte er die Wahl zwischen lokaler und systemischer Therapie sowie das therapeutische Drug-Monitoring bei sekundärem Therapieversagen bei der Colitis ulcerosa oder eine Top-down-Therapie bei Crohn-Patienten mit ungünstigen Prädiktoren bzw. einen Verzicht auf Thiopurine bei älteren Menschen.

Bisher orientiert man sich allerdings primär an klinischen Prädiktoren. Ein schwerer Verlauf eines Morbus Crohn ist z.B. zu erwarten bei perianaler Erkrankung, proximaler Ausdehnung der Erkrankung, einem Alter von $<40$ Jahren bei der Erstdiagnose und der Notwendigkeit einer initialen Steroidtherapie bzw. bei Ineffektivität von Steroiden [6]. Weiterhin haben sich NOD2-Mutationen und hohe Titer von Antikörpern gegen bakterielle Strukturen als ungünstige prädiktive Faktoren erwiesen. Für den individuellen Patienten besitzen sie jedoch wenig Aussagekraft.

$$
\text { Angelika Bischoff, Planegg }
$$

Quelle

10. TNF-alpha-Forum, Veranstalter MSD, München, 13.-14. Januar 2017

\section{Literatur \\ 1 Sandborn WJ et al: Gastroenterology 2014;146:85-95. \\ 2 Colombel JF et al.: UEGW 2013; abstr OP 260. \\ 3 Sandborn WJ et al.: Gastroenterology 2014;146:96-109. \\ 4 Colombel et al.: UEGW 2014;abstr P0890. \\ 5 Reinisch et al.: ECCO 2016;P307. \\ 6 Beaugerie et al.: Gastroenterology 2006;130:650-656.}

Weitere Informationen bei MSD SHARP \& DOHME GMBH

infocenter@msd.de

www.msd.de / www.simponi.de

\title{
PharmaTicker+++ PharmaTicker+++ PharmaTicker+++ PharmaTicker+++
}

BMS hat jetzt Ergebnisse der Studie ONO-453812 präsentiert. Diese zeigen, dass Nivolumab $\left(\right.$ Opdivo ${ }^{\circledR}$ ) bei vorbehandelten Patienten mit fortgeschrittenem Magenkarzinom, die refraktär gegenüber der Standardtherapie sind oder diese nicht vertragen, das Mortalitätsrisiko signifikant um 37\% reduziert. ONO-4538-12 ist eine randomisierte, doppelblinde, placebokontrollierte klinische Studie der Phase-III zur Beurteilung der Wirksamkeit und Sicherheit von Nivolumab bei diesen Patienten, für die es derzeit keinen Behandlungsstandard gibt.

Bristol-Myers Squibb GmbH \& Co. KGaA www.b-ms.de / www.bms-onkologie.de
Lilly. Der Angiogenesehemmer Ramucirumab $\left(\right.$ CYRAMZA $^{\circledR}$ ) wurde Anfang des Jahres 2016 EU-weit in Kombination mit FOLFIRI zur Therapie von vorbehandelten Patienten mit metastasiertem Kolorektalkarzinom (mCRC) zugelassen. Die Bedeutung dieser neuen Behandlungsoption für mCRC-Patienten spiegelt sich in der Empfehlung der ESMO Consensus Guideline mit dem höchsten Empfehlungsgrad I, A wider. In den vergangenen Jahren hat sich die Chance der Betroffenen auf eine langfristige Krankheitskontrolle erheblich verbessert.

Lilly Deutschland GmbH

www. lilly-pharma.de / www.cyramza.de
Bayer. Die US-amerikanische Gesundheitsbehörde Food and Drug Administration (FDA) hat im Zulassungsverfahren für eine Indikationserweiterung von Regorafenib den Status der vorrangigen Prüfung («Priority Review») gewährt. Das Krebsmedikament von Bayer soll zukünftig auch bei der Zweitlinienbehandlung von Patienten mit inoperablem Leberzellkarzinom zur Anwendung kommen.

Bayer AG

www.bayer.de 
Visc Med 33 | 1 | 17

Hepatische Enzephalopathie

\section{Neue Website zu XIFAXAN ${ }^{\circledR} 550$ mg jetzt online}

Auf der neuen Website www.XIFAXAN.de der Norgine $\mathrm{GmbH}$ erhalten medizinische Fachkreise ab sofort kompakte Informationen zur Diagnose und Therapie der hepatischen Enzephalopathie (HE) sowie zum Wirkmechanismus von XIFAXAN ${ }^{\circledR} 550 \mathrm{mg}$. Im Servicebereich stehen verschiedene Materialien zum Download bereit.

Dort finden Ärzte zum Beispiel eine Studienübersicht zu Rifaximin-a sowie einen Zahlenverbindungstest, ein einfaches Tool zur Diagnose der HE. Zur Aufklärung von Patienten und Angehörigen steht die Broschüre der ebenfalls von Norgine ins Leben gerufenen Initiative Lichtblicke zur Verfügung. Für Fra- gen rund um die Leber sind zudem die Adressen zahlreicher Anlaufstellen verzeichnet. Informationen zur HE und ihren Symptomen wie kognitiven und motorischen Defiziten [1] finden Besucher hier ebenso wie das Wichtigste zu Diagnostik, Krankheitsverlauf und Therapie. Für XIFAXAN ${ }^{\circledR} 550$ mg konnte gezeigt werden, dass das relative Risiko für wiederkehrende Episoden der HE signifikant sinkt $[2]^{*}$ und sich die Lebensqualität der Betroffenen verbessert [3].

Veranstaltungstermine zu medizinischen Weiterbildungen oder internationalen Kongressen mit Beteiligung von Norgine sind auf der Website ebenfalls aufgelistet. Besucher haben darüber hinaus die Möglichkeit, sich auch über XIFAXAN ${ }^{\circledR}(200 \mathrm{mg})$ zu informieren. In dieser Dosierung ist das Präparat in Deutschland bereits seit 2008 zur Behandlung der Reisediarrhö zugelassen [4].

${ }^{*} 91 \%$ der Patienten in beiden Behandlungsarmen erhielten als Begleitmedikation Lactulose.

\section{Literatur}

1 Bajaj et al.: Gastroenterology 2010;138:2332-2340.

2 Bass NM et al.: N Engl J Med 2010;362:1071-1081.

3 Sanyal A et al.: Aliment Pharmacol Ther 2011;34: 853-861.

4 Fachinformation XIFAXAN ${ }^{\circledR}$, Stand 05/2015.

Weitere Informationen bei

Norgine $\mathrm{GmbH}$

info@norgine.de

www.norgine.de / www.xifaxan.de

\section{Europäische Kommission erteiltt Marktzulassung für VEMLIDY® (Tenofoviralafenamid, TAF) von Gillead zur Behandllung der chronischen Hepatitis-B-Infelktion}

Die Europäische Kommission hat die Zulassung für Vemlidy ${ }^{\circledR}$ (Tenofoviralafenamid, TAF) 25 mg als einmal täglich einzunehmende Tablette zur Behandlung der chronischen Hepatitis-BVirus (HBV)-Infektion bei Erwachsenen und Jugendlichen (ab 12 Jahren mit einem Körpergewicht von mindestens $35 \mathrm{~kg}$ ) erteilt. Dies ermöglicht nun die Vermarktung von TAF in den 28 Ländern der Europäischen Union sowie in Norwegen und Island. In Deutschland wird Gilead Vemlidy ${ }^{\circledR}$ voraussichtlich ab 1. April 2017 zur Verfügung stellen.

«Als erste neu in Europa zugelassene Behandlung für chronische Hepatitis B seit fast einem Jahrzehnt zeigt diese Zulassung den Fortschritt in der Behandlung einer progredienten, lebensbedrohlichen Erkrankung, die 13 Millionen Europäer betrifft», erklärte Professor Pietro Lampertico, Leiter der Abteilung für Gastroen- terologie und Hepatologie am Fondazione IRCCS Ca' Granda Ospedale Maggiore Policlinico, von der Universität Mailand (Italien). «Die Behandlung einer lebenslangen Erkrankung wie der chronischen Hepatitis B kann mit zunehmendem Alter der Patienten eine Herausforderung darstellen. Die unter TAF gezeigten Verbesserungen der Labor-Sicherheitsparameter für Knochen und Niere im Vergleich zu Tenofovirdisoproxilfumarat (TDF) machen die Therapie zu einer wichtigen neuen Option für die Patienten.»

TAF ist ein neues zielgerichtetes Prodrug von Tenofovir, das im Vergleich zu TDF (Viread ${ }^{\circledR}$, Gilead Sciences) bei einer wesentlich geringeren Dosis eine ähnlich hohe Effektivität aufweist. TAF kann mit nur einem Zehntel der Dosis verabreicht werden, weil es im Plasma stabiler ist und Tenofovir effizienter zu den
Hepatozyten (Leberzellen) bringt als TDF. Damit gelangt auch weniger Tenofovir in den Blutkreislauf, weswegen TAF in klinischen Studien mit besseren Laborparametern zur Sicherheit für Nieren und Knochen assoziiert war als TDF. Die Zulassung von TAF wurde durch Daten aus 2 internationalen Phase-III-Studien (Studien 108 und 110) über 48 Wochen bei 1298 erwachsenen Patienten mit chronischer HBV-Infektion gestützt.

Weitere Informationen bei GILEAD Sciences GmbH

Daria Munsel

Daria.Munsel@gilead.com www.gilead.com

ECCO-Update zeigt zunehmende Unterstützung für Biosimilars bei CED -

Umstellung von Remicade ${ }^{\circledR}$ auf Remsima ${ }^{\circledR}$ befürrwortet

Die European Crohn's and Colitis Organisation (ECCO) hat eine aktualisierte Stellungnahme veröffentlicht, in der sie den Einsatz von Infliximab-Biosimilars für die Behandlung von chronisch-entzündlichen Darmerkrankungen (CED) befürwortet [1]. Die Fachgesellschaft betont, dass Infliximab-Biosimilars vergleichbar wirksam und sicher wie das Referenzprodukt sind, und unterstützt die Umstellung der Patienten vom Originator auf Remsima ${ }^{\circledR}[1]$. Die Stellungnahme der ECCO wurde im Journal of Crohn's and Colitis veröffentlicht [1]. Mit dem neuen Positionspapier vollzieht die ECCO einen Richtungswechsel gegenüber früheren Empfehlungen, die ein vorsichtigeres Vorgehen befürworteten [2]. Die positive Stellungnahme für den Einsatz von Biosimilars bei CED spiegelt sowohl die zunehmende Evi- denz als auch das gestiegene Vertrauen von Ärzten in diese Substanzen wider $[1,3]$.

Literatur

1 Danese S et al.: J Crohns Colitis 2017;11:26-34.

2 Danese S et al.: J Crohns Colitis 2013;7:586-589.

3 Danese S et al.: J Crohns Colitis 2016;10:1362-1365.

Mundipharma Deutschland GmbH \& Co. KG

info@ mundipharma.de

www.mundipharma.de 
Visc Med $33|1| 17$

\section{8-wöchige Therapie mit VIEKIRAX ${ }^{\circledR}+$ EXVIERA ${ }^{\circledR}$ erzielt in alktueller Studie eine SVR $_{12}$ von $98 \%$ bei Hepatitis-C-Patienten vom Genotyp 1b}

AbbVie gibt neue Daten bekannt, die sehr hohe Heilungsraten bei einer nur 8-wöchigen Behandlung mit VIEKIRAX ${ }^{\circledR}$ (Ombitasvir/ Paritaprevir/ Ritonavir) + EXVIERA ${ }^{\circledR}(\mathrm{Da}-$ sabuvir) belegen. In der Phase-IIIb-Studie GARNET erreichten $98 \%\left(\mathrm{n}=160 / 163^{*}\right)$ der nicht vorbehandelten Patienten mit chronischer Hepatitis-C-Virus (HCV)-Infektion vom Genotyp 1b (GT1b) ohne Leberzirrhose ein anhaltendes virologisches Ansprechen 12 Wochen nach Therapieende (sustained virologic response, $S_{V R}$ ) [1]. Diese Daten wurden bei der European Association for the Study of the Liver (EASL) Special Conference 2016 New Perspectives in Hepatitis C Virus Infection The Roadmap for Cure in Paris vorgestellt und sind in den neu veröffentlichten Empfehlungen der EASL zur Therapie der chronischen Hepatitis C enthalten. VIEKIRAX + EXVIERA ist derzeit in der Europäischen Union für die Behandlung von GT1b-Patienten mit und ohne kompensierte Zirrhose (Child-Pugh A) über 12 Wochen zugelassen. «Unter 12-wöchiger Behandlung mit
VIEKIRAX + EXVIERA werden bereits sehr hohe Heilungsraten verzeichnet», so Prof. Stefan Zeuzem, Studienautor und Direktor der Medizinischen Klinik am Universitätsklinikum der J. W. Goethe-Universität in Frankfurt am Main. «Die Ergebnisse belegen nun, dass auch eine nur 8-wöchige Therapie mit VIEKIRAX + EXVIERA zu einer Heilung der Hepatitis C führen kann. Diese Ergebnisse sind insofern besonders bedeutsam, als dass sie sich auf Patienten mit dem am weitesten verbreiteten HCV-Genotyp beziehen.»

Etwa 160 Millionen Menschen sind weltweit mit HCV infiziert [2]. GT1 ist der häufigste der 6 HCV-Genotypen, von dem weltweit geschätzte 83 Millionen Menschen betroffen sind [3]. In Europa ist GT1b der vorherrschende Genotyp, der $47 \%$ der 9 Millionen chronischen HCV-Fälle ausmacht [3-5]. Von den HCVPatienten in Deutschland sind rund 38,4\% mit dem GT1b infiziert [6].

«AbbVies Fokus ist, die Bedürfnisse im Rahmen der HCV-Versorgung zu identifizieren und ihnen nachzukommen, dazu zählt auch die Verkürzung der Behandlungsdauer», erklärte Dr. Rob Scott, Vice President, Development und Chief Medical Officer von AbbVie.

${ }^{*}$ mITT-GT; 3 Patienten, die nicht den GT1b aufwiesen, wurden bei der Analyse ausgeschlossen.

Literatur

1 Welzel T et al.: GARNET: High SVR Rates Following Eight-Week Treatment with Ombitasvir/Paritaprevir/ Ritonavir + Dasabuvir for Patients with HCV Genotype $1 \mathrm{~b}$ Infection. Presented at the European Association for the Study of the Liver Special Conference: New Perspectives in Hepatitis C Virus Infection - The Roadmap for Cure, Paris, France on September 23-24, 2016.

2 Lavanchy D: Clin Microbiol Infect 2011;17:107-115.

3 Messina JP et al.: Hepatology 2015;61:77-87.

4 O'Leary JG et al.: Hepatitis C; in Feldman M, Friedman LS, Brandt LJ (eds): Sleisenger and Fordtran's Gastroin testinal and Liver Disease: Pathophysiology/Diagnosis/ Management, ed 9, vol 1. Philadelphia, Saunders Elsevier, 2010, pp 1313-1335.

5 Hatzakis A et al.: J Viral Hepat 2011;18(suppl 1):1-16. 6 Hüppe D et al.: J Viral Hepat 2015;22:22.

Weitere Informationen bei AbbVie Deutschland GmbH \& Co. KG info.de@abbvie.com

www.abbvie.de

Neuer Service von Takeda jetzt verfügbar

\section{Das Patientensupportprogramm myvyo ${ }^{\mathrm{TM}}$ mit individualisiertem Coaching-Konzept für} CED-Patienten mit Vedolizumab (Entyvio ${ }^{\circledR}$ )-Therapie

Die Diagnose einer chronisch-entzündlichen Darmerkrankung (CED) bedeutet für die $\mathrm{Pa}$ tienten einen großen Einschnitt in ihr Leben. Viele Fragen bewegen die Betroffenen mit Morbus Crohn oder Colitis ulcerosa: zum Verlauf der Erkrankung, zu Therapieoptionen, aber auch zu damit verbundenen Belangen des täglichen Lebens. Den Patienten Gehör schenken, ihre Ängste und Befürchtungen ernst nehmen, ihre Bedürfnisse kennenlernen und sie möglichst in vielen gewünschten Bereichen ihres Alltags unterstützen - das sind die Anforderungen an ein gutes Patientenprogramm. Diesen Vorgaben entsprechend wurde von Takeda das myvyo ${ }^{\mathrm{TM}}$-Programm für eine umfassende Unterstützung von Vedolizumab (Entyvio $\left.{ }^{\circledR}\right)$-Patienten entwickelt. myvyo ${ }^{\text {TM }}$ hält mit seinem einmaligen Coaching-Konzept eine individualisierte unterstützende Begleitung für die mit Vedolizumab (Entyvio ${ }^{\circledR}$ ) behandelten Morbus Crohn- bzw. Colitis ulcerosa-Patienten bereit. Die Teilnehmer können zudem die zahlreichen Informationsangebote auf dem myvyo ${ }^{\mathrm{TM}}$-Online-Portal nutzen. Ulrike Talkenberg, Patient Care Manager CED bei Takeda, erläuterte das Grundgerüst des myvyo ${ }^{\mathrm{TM}}$-Patientenprogramms: Der Teilnehmer erhält - auf Wunsch - eine individuell auf ihn zugeschnittene Beratung durch einen speziell ausgebildeten Coach, vor allem zu Fragen des individuellen Lebensstils mit der Erkrankung. Relevante Themen können hier zum Beispiel Stressmanagement, eine passende Ernährung oder die sportliche Betätigung sein. Der persönliche Austausch mit dem Coach zu solchen und vielen weiteren Themen kann telefonisch oder online erfolgen. Den zweiten Baustein des Programms stellt das myvyo ${ }^{\mathrm{TM}}$-Online-Portal dar. Hier können die Teilnehmer - auch ohne aktiven Kontakt zu einem Coach - zahlreiche Informationsangebote rund um Colitis ulcerosa und Morbus Crohn nutzen sowie eine Selbsteinschätzung ihres Wohlbefindens vornehmen und persönliche Veränderungen verfolgen. Bereits seit der Pilotphase begleitet Jennifer Zemke das myvyo ${ }^{\mathrm{TM}}$-Programm. Sie ist 2. Vorsitzende der Fachgesellschaft für Assistenzpersonal CED (FA CED e.V.) und seit vielen Jahren als Versorgungsassistenz CED und Study Nurse in einer gastroenterologischen Praxis in Herne tätig. Es habe sich ge- zeigt, dass dieses Patientenprogramm gut in den Praxisalltag zu integrieren sei: Direkt nach der ärztlichen Entscheidung zur Vedolizumab (Entyvio ${ }^{\circledR}$ )-Therapie informiere sie den Patienten über das myvyo ${ }^{\mathrm{TM}}$-Programm, und am Tag der ersten Infusion werde ihm das Starter-Kit ausgehändigt. "myvyo ${ }^{\mathrm{TM}}$ hat sich bisher als nützlicher zusätzlicher Baustein in der Betreuung von CED-Patienten erwiesen. Es bietet viele Optionen der Unterstützung, die jeder für sich individuell wählen und nutzen kann», schloss Zemke.

\section{Quelle}

Takeda Pressegespräch «Der CED-Patient zwischen Informationsbedürfnis, Therapieoptionen und Alltag - welche Unterstützung können moderne Wege in der Patientenkommunikation bieten?», 20. Januar 2017, Köln

Weitere Informationen bei Takeda Pharma Vertrieb GmbH \& Co. KG medinfo@takeda.de www.takeda.de / www.myvyo.com 
Visc Med 33 | 1 | 17

\section{Ocaliva ${ }^{\circledR}$ zur Behandllung der primär biliären Cholangitis zugelassen}

Ein rund 20-jähriger Innovationsstau hat sein Ende gefunden. Kürzlich wurde Ocaliva ${ }^{\circledR}$ (Obeticholsäure (OCA)) zur Behandlung der primär biliären Cholangitis (PBC) in der EU zugelassen [1], sodass in dieser Indikation nun eine neue Therapieoption zur Verfügung steht. In der Phase-III-Studie POISE erzielte OCA bei Patienten, die auf Ursodesoxycholsäure (UDCA) unzureichend ansprachen oder diese nicht tolerierten, signifikant bessere Ansprechraten als auf UDCA alleine bzw. mit Placebo [2]. Damit schließt das Medikament eine therapeutische Lücke. Denn bisher gab es für PBC-Patienten, die mit UDCA nicht adäquat behandelt werden konnten, keine weitere $z u$ gelassene Option.

Die PBC ist eine autoimmun vermittelte Lebererkrankung, bei der es zu einer Entzündung der intrahepatischen Gallenwege und nachfolgend $\mathrm{zu}$ einer Cholestase kommt. «Unbehandelt droht ein fibrotischer Umbau des Lebergewebes bis hin zu Leberzirrhose, Transplantationsbedarf und leberbedingtem Tod», sagte Prof. Dr. Dr. Michael Kraus, Altötting [3]. In Deutschland sind schätzungsweise zwischen 4000 und 12000 Menschen an einer PBC erkrankt, wobei in 90\% aller Fälle Frauen betroffen sind [4]. Die Standardbehandlung bestand bislang aus der Gabe von UDCA. Sie verbessert die klinische Symptomatik, verlangsamt die Progredienz und erhöht die Überlebenswahrscheinlichkeit $[3,5]$. Allerdings sprechen bis zu $40 \%$ der Patienten nicht adäquat auf UDCA an [6], andere tolerieren das Medikament nicht. «Da es bis vor kurzem keine zugelassene Alternative gab, hatten diese Patienten ein erhöhtes Risiko für ein Fortschreiten der Erkrankung, für Leberzirrhose und vorzeitigen Tod», erläuterte Kraus $[2,4,5,7]$. Nun steht mit Ocaliva ${ }^{\circledR}$ eine wirksame und verträgliche neue Substanz zur Verfügung.

«OCA ist ein Agonist des nukleären FarnesoidX-Rezeptors (FXR), der primär in Leber und Darmtrakt gebildet wird», erklärte Prof. Dr. Thomas Berg, Leipzig [2].«Eine Aktivierung des Signalwegs führt dazu, dass Gallensäuren vermindert synthetisiert und verstärkt sezerniert werden» [2]. Dadurch schützt OCA die Hepatozyten vor dem toxischen Einfluss der Gallensäuren. Darüber hinaus führt eine Aktivierung des FXR-Signalwegs vermutlich zu antiinflammatorischen und antifibrotischen Effekten [2].

In der randomisierten und doppelblinden Phase-III-Studie POISE wurde OCA bei PBC-Patienten geprüft, die auf UDCA nicht ausreichend angesprochen hatten $(\mathrm{n}=200)$ oder das Medikament nicht tolerierten ( $\mathrm{n}=16)$. «Zusammengefasst zeigt die POISEStudie, dass eine 12-monatige Behandlung der PBC mit OCA zusätzlich zu UDCA der Gabe von UDCA alleine bzw. Placebo überlegen ist und die Verbesserungen bei biochemischen Krankheitsmarkern über 2 Jahre anhalten», resümierte Berg. Dabei wurde die Therapie im Allgemeinen gut vertragen [2]. Auf Grundlage der POISE-Studie wurde OCA zur Behandlung Erwachsener mit PBC zugelassen [8]. «Patienten, die unzureichend auf UDCA ansprechen, sollen Obeticholsäure kombiniert mit UDCA erhalten - Patienten, die UDCA nicht tolerieren, als Monotherapie», erläuterte Dr. Dr. Andreas Kremer, Erlangen [8]. «Die Anfangsdosis beträgt einmal täglich $5 \mathrm{mg}$. Nach 6 Monaten soll die Verträglichkeit evaluiert und die Dosierung in Abhängigkeit davon auf $10 \mathrm{mg}$ täglich erhöht werden [8].» Die POISE-Studie hatte gezeigt, dass sich durch dieses Vorgehen unter Erhaltung der Effektivität die Abbruchrate auf $1 \%$ reduzieren ließ [2]. In diesem Zusammenhang verwies Kremer auch darauf, dass die in der POISE-Studie initial erhöhte Pruritus-Belastung unter Verum im Verlauf zurückging und nach 12 Monaten der unter Placebo glich [2].

\section{Quelle}

Launch-Pressekonferenz «Ocaliva ${ }^{\circledR}$ - eine neue Behandlungs-Perspektive für Patienten mit primär biliärer Cholangitis»; eine Veranstaltung der Intercept Pharma Deutschland GmbH

\section{Literatur}

1 www.ema.europa.eu/ema/index.jsp?curl=pages/ medicines/human/medicines/004093/human med_002043.jspermid=WC0b01ac058001d124 (letzter Zugriff am 16. Januar 2017).

2 Nevens F et al.: N Engl J Med 2016;375:631-643.

3 Poupon et al.: J Hepatol 2010;52:745-758.

4 www.leberhilfe.org/pbc.html (letzter Zugriff am 16. Januar 2017).

5 Lindor KD et al.: Hepatology 2009;50:291-308.

6 Lammers WJ et al.: Gastroenterology 2014;147: 1338-1349.

7 Selmi C et al.: Lancet 2011;377:1600-1609.

8 Fachinformation Ocaliva ${ }^{\circledR}$, Stand Dezember 2016.

\section{Weitere Informationen bei}

Intercept Pharma Deutschland GmbH

Dr. Diane Langenbacher

diane.langenbacher@interceptpharma.com
Beschleunigtes Zulassungsverfahren für AbbVies Glecaprevir/Pibrentasvir (G/P) zur Behandlung der chronischen Hepatitis C aller Genotypen

AbbVie gibt bekannt, dass die Europäische Arzneimittel-Agentur den Zulassungsantrag für das sich in der Prüfphase befindliche Therapieregime Glecaprevir/Pibrentasvir (G/P) zur Behandlung von Patienten mit chronischer Hepatitis C aller Genotypen (GT1-6) angenommen hat und ein beschleunigtes Zulassungsverfahren gewährt. Bei Zulassung von G/P stünde therapienaiven chronischen Hepatitis-C-Patienten aller Genotypen (GT1-6) ohne Leberzirrhose eine 8-WochenTherapie zur Verfügung. Diese Patientengruppe bildet die Mehrheit der heutigen $\mathrm{He}-$ patitis-C-Patienten. Darüber hinaus stünde bei Zulassung von G/P für Patienten mit kompensierter Leberzirrhose (Child-Pugh A) eine zusätzliche Therapieoption bereit. G/P wurde zudem für die Behandlung von bislang schwer behandelbaren Patientengruppen entwickelt. Dazu zählen Patienten, die durch eine Therapie mit direkt antiviral wirksamen Substanzen (direct acting antiviral agents, DAAs) nicht geheilt wurden, oder $\mathrm{Pa}$ tienten mit schwerer bzw. terminaler Nierenfunktionsstörung, inklusive Dialyse.

AbbVie Deutschland GmbH \& Co. KG www.abbvie.de

Monoklonaler Antikörper gegen

Clostridium difficile Toxin B zugelassen

Die Europäische Kommission hat im Januar die Zulassung für den humanen monoklonalen Antikörper Bezlotoxumab (ZINPLAVA ${ }^{\circledR}$ ) erteilt. Bezlotoxumab ist als erster Wirkstoff zur Prävention der Rekurrenz einer Clostridium difficile-Infektion (CDI) bei erwachsenen Patienten mit einem hohen Rekurrenzrisiko für eine $\mathrm{CDI}$ zugelassen. Bezlotoxumab wird während der antibakteriellen Therapie gegen eine CDI als intravenöse Einmalinfusion gegeben. Basis für die Zulassung sind die 2 Phase-III-Studien MODIFY I und II. Bezlotoxumab bindet mit hoher Affinität an Clostridium difficile Toxin B und neutralisiert dessen Aktivität. Bezlotoxumab verhindert die Rekurrenz einer CDI mittels passiver Immunität gegen Toxine, die nach dem Auskeimen persistierender oder neu erworbener Clostridium difficileSporen gebildet werden. Toxin B kann die Darmwand schädigen und eine Entzündung verursachen, die meist mit Symptomen wie wässrigem Durchfall und abdominellen Schmerzen einhergeht. Es wird derzeit geprütt, ob ZINPLAVA ${ }^{\circledR}$ in Deutschland als reines Klinikprodukt vertrieben wird oder auch für den niedergelassenen Bereich relevant ist.

MSD SHARP \& DOHME GMBH

www.msd.de 